\title{
Maternal Death Surveillance System Evaluation at Addis Ababa City Administration, Ethiopia, 2018
}

\author{
Kefyalew Amene Bogale ${ }^{1,}$, , Tesfahun Abye Meshesha ${ }^{1}$, Abiy Girmay ${ }^{2}$ \\ ${ }^{1}$ Deepartment of Public Health Emergency Management, Ethiopian Public Health Institute, Addis Ababa, Ethiopia \\ ${ }^{2}$ World Health Organization, Addis Ababa, Ethiopia
}

Email address:

kefyalewamene@gmail.com (K. A. Bogale), tesfahunabye1900@gmail.com (T. A. Meshesha), abiygirmay2009@gmail.com (A. Girmay)

${ }^{*}$ Corresponding author

\section{To cite this article:}

Kefyalew Amene Bogale, Tesfahun Abye Meshesha, Abiy Girmay. Maternal Death Surveillance System Evaluation at Addis Ababa City Administration, Ethiopia, 2018. Journal of Cancer Treatment and Research. Vol. 8, No. 4, 2020, pp. 64-73. doi: 10.11648/j.jctr.20200804.11

Received: July 21, 2020; Accepted: August 4, 2020; Published: November 23, 2020

\begin{abstract}
Introduction: Public health surveillance is an ongoing, systematic collection, analysis, interpretation, and dissemination of data regarding a health-related event for use in public health. Maternal Death Surveillance and Response (MDSR) is form of continuous surveillance linking the health information system to quality improvement processes from local to national levels. Ethiopia has been implementing Maternal Death Surveillance and Response for the last four years. The aim of evaluating Maternal Death Surveillance and Response system at Addis Ababa City Administration was to evaluate the performance and identify gaps of existing system in city administration 2018. Methods: A descriptive cross-sectional study design was used to evaluate Maternal Death Surveillance and Response system at Addis Ababa City Administration, which was conducted from March 12-23/2018. Purposive sampling technique was used to select 13 study units. Primary data was collected using structured questionnaire and crosschecked with available documents. Data was analyzed using Microsoft Office Excel, 2016. Result: Only $67 \%$ of study units had appropriate denominator and the denominator at city level was 1168157 (34.6\% of total population). All study units had maternal death review committee and the action threshold used by all study units was one maternal death. A total 52 maternal death notifications received by the region from Sept/2016 to Aug/2017, giving case detection rate of $13 \%$ of national plan for the city. All notifications were true maternal deaths. Cause of death identified for 50 ( $96.15 \%)$ of deaths and only findings from 7 (14\%) deaths used for action. No separate budget was allocated for Maternal Death Surveillance and Response system at all levels. MDSR system implementation at private health facilities was almost neglected and overall average attribute measurement for the system was 63\%. Conclusion: Maternal Death Surveillance and Response system establishment objectives will not be achieved by current level of implementation and detection. Data utilization and attributes value was very low. Lack of separate budget affects the system implementation.
\end{abstract}

Keywords: Maternal Death Surveillance and Response System, Evaluation, Addis Ababa, Ethiopia 2018

\section{Introduction}

Surveillance is the process of gathering, analyzing, and dissemination of information for the purpose of proper planning, implementation, and evaluation of health services/interventions. It is also defined as "Information for Action". A functional disease surveillance system is essential for defining problems and taking action. Proper understanding and use of this essential epidemiological tool (public health surveillance) helps health workers at the woreda and health units to set priorities, plan interventions, mobilize and allocate resources, detect epidemics early, initiate prompt response to epidemics, and evaluate and monitor health interventions. It also helps to assess long term disease trends [1].

Ultimately, the purpose for conducting public health surveillance is to predict and prevent epidemics, detect epidemics/outbreaks, treat and control priority diseases, evaluate an intervention, learn and monitor the ongoing pattern of priority diseases occurrence and the potential for disease in a population so that we can be effective in investigating, controlling, and preventing disease in that population and to link with public health action [1]. 
Ethiopia had introduced Integrated Disease Surveillance and Response (IDSR) in 1996, focusing on 17 priority communicable diseases. Two years later, in 1998, the 48th WHO Regional committee for Africa adopted a resolution on integrated disease surveillance of communicable diseases. It is aimed to assist health workers to detect and respond to diseases of epidemic potential, diseases of public health importance, and diseases targeted for eradication and/or elimination through the available and effective control and prevention methods [2].

In integrated disease surveillance, the various surveillance activities become integrated into one system within the broader national health system. Recently federal ministry of health $(\mathrm{FMoH})$ underwent the process reengineering, identifying the IDSR to be the core process to be evaluated. The IDSR was evaluated and identifying its strength and weakness, was recommended to establish Public Health Emergency Management (PHEM) as of 2009. One of the major activities of PHEM is to take over the disease's surveillance parallel to preparedness, response and rehabilitation in any health-related emergencies and outbreaks [1].

The public health system is continually challenged by recurrent and unexpected disease outbreaks and is facing the challenge of managing health consequences of natural and man-made disasters, emergencies, crises, and conflicts. These problems continue to disrupt the health care system, while successful detection and response to these challenges is becoming increasingly complicated [3].

Since conducting surveillance for all health problem consumes time and other resources, the National Public Health Emergency Management Center (NPHEMC) identified 22 priority diseases and events based on their Public health importance, potential to cause outbreaks, disease of international concern, diseases on eradication or elimination, availability of control and prevention measures, and capacity of the health system to implement available control and prevention measures for the health problem. Additional to the 22 prioritized diseases and events, PHEM is also monitoring any cluster of cases and any unusual presentations. Out of these 22 prioritized diseases and events 14 are weekly reportable while 8 are immediately reportable. Maternal death is grouped in immediately reportable health related event. Regions of the country can add their own priority diseases/events in their priority disease list $[1,4]$.

Maternal Death Surveillance and Response (MDSR) is relatively new surveillance system linking the health information system and quality improvement processes from local to national levels. It includes the routine identification, notification, quantification, and determination of causes with the aim of providing information that effectively shows the true magnitude of maternal mortality and guides actions to eliminate preventable maternal mortality at a health facilities and community level [5].

According to WHO technical guide line for MDSR, there are four basic processes/activities in the implementation of MDSR system. These basic activities are Identification and notification of a suspected maternal death (community or facility death), Review of maternal death by local maternal death review committee, analysis and interpretation of aggregated findings from the review and finally implement and monitor recommendations made by the review committee [5].

During the last two decades, maternal mortality level in Ethiopia reduced by $71 \%$ from its level in 1990 (1250/100,000 live births to $353 / 100,000$ live births in 2015). However, this achievement still short of the country's target to reach 267/100,000 live births by 2015 [6, 7]. According to the 2016 Ethiopian DHS, maternal mortality ratio (MMR) is around 412/100,000 live births [8].

The government of Ethiopia has developed the five-year (2016 to 2020) health sector transformation plan (HSTP) and RH strategy for 2016-2020, putting reduction of maternal and perinatal deaths as a top priority [9]. WHO and other partners are on the process of implementing this new approach towards maternal death specifically on 75 high-burden countries that account for $95 \%$ of maternal deaths [10].

Ethiopia is one of the countries with a higher number of maternal deaths. There has been effort in the country to improve access of essential health service to the population with specific focus on mothers and children. Following this, Ethiopia has been implementing MDSR for the last four years to address preventable maternal deaths following the 2013 WHO technical guidance. The national MDSR database receives reports and case summaries from all regions in the country $[11,12]$.

The main aim of establishing MDSR system is to provide accurate information on the magnitude of maternal deaths and about preventable factors that contribute to a maternal death which attracts attention of policy and decision makers, and guides actions that need to be taken at the each level and assess the effectiveness of these actions to prevent maternal deaths. To achieve its aim, MDSR system uses community and facility-based surveillance system [12].

For a maternal death happening at a community the HEWs will collect and compile detail information about the event by interviewing key informants such as local Health Development Army (HAD), religious leaders, administrative leaders, community members and family members. Any of these individuals report a death of a woman of reproductive age (15-49 years) to HEWs. The HEWs intern notify to her supervisor, and the supervisor report to a health facility. MDSR review committee at that health facility will arrange verbal autopsy (VA) to be conducted. The MDSR review committee of the health facility will complete the VA by interviewing individuals who attended the women during illness, in Laboure and delivery (for home delivery), a Person who were present at the time of death and any person who is likely to have additional details on the woman's experiences during pregnancy such as husband and children [12].

For a maternal death happening at a health facility, MDSR review committee use referral sheets, medical records, attending health workers (OPD, maternity ward, operation 
room) and Log books to complete verbal autopsy. To do all maternal reviews, a consent after informed decision of the participants need to be obtained and confidentiality should be maintained. The national guideline for MDSR recommends to use different information dissemination methods, starting from presentation on meetings to scientific publications and audio-visual reports, ensuring that the information gets to reach the right audience (who can act on the recommendations) [12].

WHO estimates show that the leading causes of maternal deaths in the world are hemorrhage and hypertension, which together account for more than half of all maternal deaths. Indirect causes, which include deaths due to conditions such as Malaria, HIV/AIDS and cardiac diseases, account for about one fifth of maternal deaths [3].

In developing countries hemorrhage and hypertension are among the top three causes of deaths in both South Asia and Sub-Saharan Africa. The majority of maternal deaths in developing countries are due to five major direct obstetric complications: hemorrhage, infection, unsafe abortion, hypertensive disorders of pregnancy, and obstructed Laboure $[13,14]$.

Like any other developing countries, Ethiopia is also affected by the above causes of maternal mortality. A systemic review conducted by Yifru Berhan and Asres Berhan on eight facility based maternal death studies conducted from 1980 to 2012 indicates that the top four causes of maternal mortality in the year 1980-1999 were abortion related complications (31\%), obstructed labor/uterine rupture (29\%), sepsis/infection $(21 \%)$ and hemorrhage $(12 \%)$. In the last decade, however, the top four causes of maternal mortality were obstructed labor/uterine rupture $(36 \%)$, hemorrhage (22\%), hypertensive disorders of pregnancy (19\%) and sepsis/infection (13\%) [15].

Maternal death is a very complex event to understand the contribution and association among risk factors of it. These complex risk factors can be categorized in to biological factors (age, parity, birth spacing), socio economic factors, health system and health facility associated factors [16].

Public health surveillance systems should be evaluated periodically, and the evaluation should include recommendations for improving quality, efficiency, and usefulness. Evaluation of a public health surveillance system focuses on how well the system operates to meet its purpose and objectives. The evaluation of public health surveillance systems should involve an assessment of system attributes, including simplicity, flexibility, data quality, acceptability, sensitivity, predictive value positive, representativeness, timeliness, and stability. Because public health surveillance systems vary in methods, scope, purpose, and objectives, attributes that are important to one system might be less important to another. A public health surveillance system evaluation should emphasize those attributes that are most important for the objectives of the system [17].

It is important to monitor data quality and thus ensure that the collected data are meaningful so they meet the objectives of local, national and international surveillance systems. The quality of the initial data may determine the data quality at all stages of the reporting process. Monitoring data quality also helps to improve data analysis and interpretation in public health reports at all levels. The purpose of evaluating maternal death surveillance system at Addis Ababa City Administration was to ensure that maternal death is being monitored effectively and efficiently with quality data provision and utilization to achieve its establishment objectives.

\section{Objective}

\subsection{General Objective}

The general objective of evaluating maternal death surveillance system at Addis Ababa City Administration was:

a) To evaluate the performance of existing maternal death surveillance system at Addis Ababa City Administration 2018.

b) To provide MDSR related information for better improvement of the system at Addis Ababa City Administration 2018.

\subsection{Specific Objectives}

The specific objectives of evaluating maternal death surveillance system at Addis Ababa City Administration, March 2018 were:

a) To assess the performance of MDSR system in relation to death detection, reporting.

b) To identify gaps and challenges in implementing the system (logistic, training, supervision and feedback).

c) To determine whether information generated from the system are used for action.

d) To evaluate the maternal death surveillance system attributes.

\section{Methods}

\subsection{Study Area and Population}

This MDSR system evaluation was carried out at Addis Ababa City Administration (capital city of Ethiopia). The city has been divided in to ten sub-cities and 116 woredas. The population of Addis Ababa was projected to be $3,433,999$ based on 2007 population enumeration with yearly growth rate of $3.8 \%$ and population density of $5,165 / \mathrm{km}^{2}$. The population of the city administration is increasing due to increased migration of peoples from rural areas to the city. The total area of the city administration is $530.14 \mathrm{~km}^{2}$. The study populations were all females in the age group of 15 to 49 years. The city had an international airport which enables peoples in the world to contact within hours of travel $[18,19]$. 


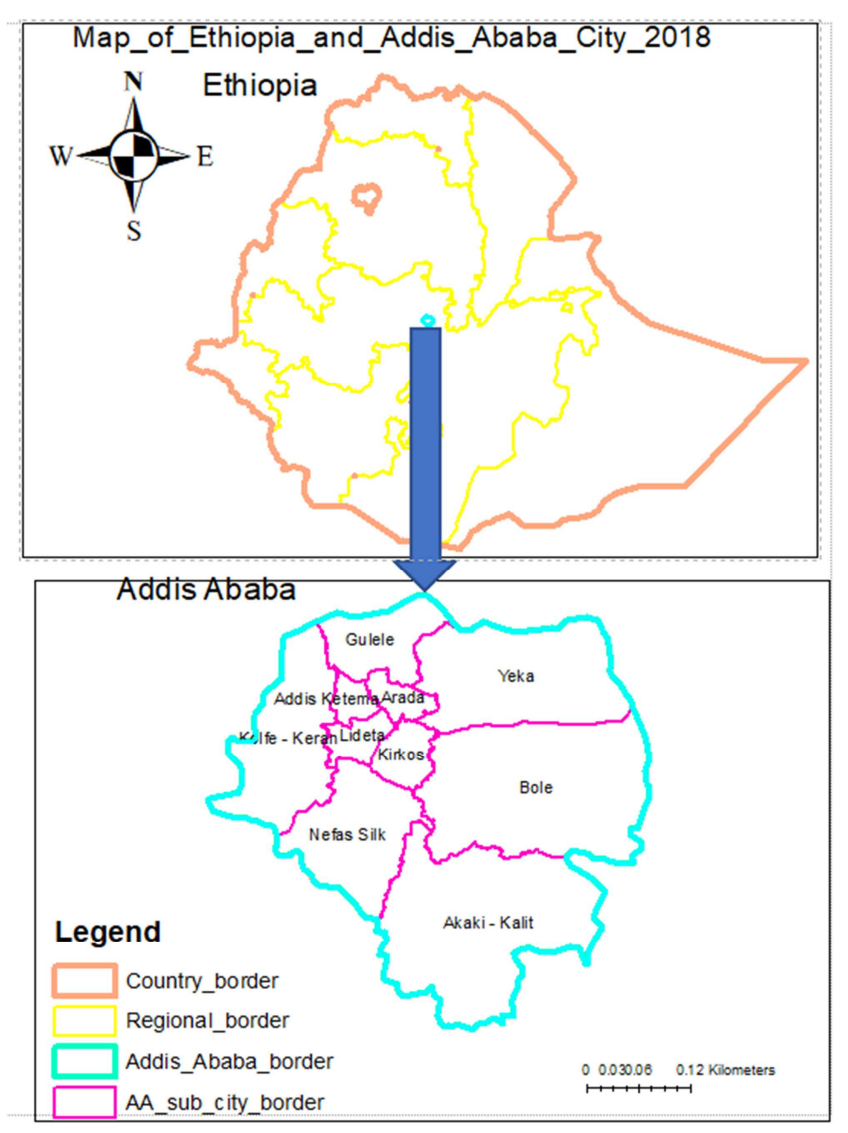

Figure 1. Map of Ethiopia and Addis Ababa, 2018.

\subsection{Study Design and Period}

A descriptive cross-sectional study was conducted at Addis Ababa City Administration. This maternal death surveillance and Response system evaluation project was carried out from March 12-23/2018.

\subsection{Sampling Technique}

Purposive sampling technique was used to select two sub-cities (Kolfe Keraniyo and Nefas silk sub-cities) out of ten (10) sub-cities found in the city administration of Addis Ababa. In communication with the City Administration PHEM (MDSR focal person), one best performing sub-city and one least performing Sub-city was selected. Similarly, two woredas and two hospitals from each sub-city was selected. Lastly one health center from each woreda was selected. Since there was no government hospital in Nefas silk sub-city, S.t Paul Hospital (with high maternal death report) from Gulele sub-city was included. Finally, data was collected from 13 study units (the regional PHEM, two sub-city PHEM, four woreda PHEM, four health centers and two hospitals).

\subsection{Study Units}

The study subjects were Addis Ababa City Administration Health Bureau Public Health Emergency Management (PHEM) unit, Kolfe Keraniyo and Nefas silk sub-cities PHEM unit, selected four woredas PHEM unit and selected two hospitals and four health facilities.

\subsection{Data Collection Tool and Method}

A structured questioner used for evaluating maternal death surveillance and response system in another previous study was used with some amendment. Pertinent primary data to evaluate MDSR system was collected from each study participants by using pre-prepared questioner. Secondary data from available documents related to maternal death surveillance was also reviewed and used to cross check with the primary data. MDSR related reports and guidelines were also observed.

\subsection{Data Quality Control and Analysis}

Data collected at each level was crosschecked with each other and data available at national level. It was also cross checked with any available document (hard copy and soft copy) related to maternal death at each level. The completeness of the data variables was checked for most of the respondents. Observation was also conducted to confirm some responses like availability of reports. The collected raw data was entered and analyzed using Microsoft Office Excel, 2016.

\subsection{Ethical Clearance}

An official letter was submitted to Addis Ababa City Administration Health Bureau PHEM unit which was cascaded to selected Sub-cities, woredas and health facilities to get permission for the evaluation.

\subsection{Operational Definition}

Acceptability: Acceptability reflects the willingness of persons and organizations to participate in the surveillance system [20].

Active surveillance system: is a system that is 'based on the public health officials' initiative to contact the physicians, laboratory or hospital staff or other relevant sources to report data [21].

Completeness: proportion of all expected data reports that were actually submitted to the public health surveillance system [21].

Comprehensive surveillance systems: include reports of cases of infectious diseases that occur within the whole population of the geographical area covered by the surveillance system [21].

Data Quality: the completeness and validity of the data recorded in the public health surveillance system [21].

Direct Obstetric Deaths: Deaths resulting from obstetric complications of the pregnant state (pregnancy, Laboure and puerperium), from interventions, omissions, incorrect treatment, or from a chain of events resulting from any of the above [12, 5].

Flexibility: A flexible public health surveillance system can adapt to changing information needs or operating conditions with little additional time, personnel, or allocated funds [20].

Indirect Obstetric Deaths: Deaths resulting from previous existing disease or disease that developed during pregnancy and which was not due to direct obstetric causes, but which was aggravated by physiologic effects of pregnancy $[12,5]$. 
Maternal Mortality Rate: The number of registered deaths among women, from any cause related to or aggravated by pregnancy or its management (excluding accidental or incidental causes) during pregnancy, childbirth or within 42 days of termination of pregnancy, irrespective of the duration or site of the pregnancy, for every 100000 live births in a given year or period of time [22].

Passive surveillance: relies on the physicians, laboratory or hospital staff or other relevant sources to take the initiative to report data to the health department [21].

Population under surveillance: defined as targeted groups (risk groups) which is determined according to objectives of the surveillance system and should take into account feasibility [21].

Representativeness: describes the occurrence of a health-related event over time and its distribution in the population by place and person [20].

Sensitivity: The sensitivity of the surveillance system is the number of cases reported by the surveillance system or 'true cases' (a), divided by the number of cases $(a+c)$ in the community [23].

Simplicity: The simplicity of a public health surveillance system refers to both its structure and ease of operation, while still meeting their objectives [20].

Stability: refers to the reliability and availability of the public health surveillance system [21].

Timeliness: reflects the speed between steps in a public health surveillance system [20].

Usefulness: Implies that surveillance results are used for public health action [20].

\section{Result}

The respondents were in the position of Regional MDSR focal person, disease prevention case team coordinator, sub-city PHEM officers, woreda PHEM officers and health facility surveillance focal persons. MDSR system implementation was launched in May 2013 by FMOH as stand-alone parallel data collection and analysis system by $\mathrm{MCH}$ department with overall goal of improving quality of care and elimination of preventable maternal deaths. MDSR was integrated into the existing Public Health Emergency Management (PHEM) system and has been added as the 21st national notifiable diseases and conditions in 2014 .

\subsection{General Information}

Sixty- seven percent (67\%) of study units had appropriate denominator for MDSR system implementation. The population included in Maternal Death Surveillance and Response (denominator) in the city administration of Addis Ababa were all women in the age group of 15 to 49 years. The appropriate denominator for the system at the regional level was 1188164 (34.6\% of the total population). The common type of surveillance used for MDSR in the city administration was comprehensive passive surveillance. The national PHEM guideline and MDSR guideline were available at $91.67 \%$ and $92.31 \%$ of study units, respectively. All other recommended formats for MDSR were available at all reporting levels.
Although the region didn't lack any of the recommended formats for MDSR in the last year (2009 EC), 33.33\% of health facilities lacked clinical register for maternal death. The action threshold used for MDSR at all study units was one maternal death and $69.23 \%$ of study units had the capacity to respond for a maternal death within 48 hours. Lack of timely notification, poor coordination and communication and shortage of supply were raised as an obstacle for providing timely response, within 48 hours. Generally, MDSR system implementation at private health facilities was almost neglected.

\subsection{Framework of MDSR Implementation}

Although everybody is expected to report maternal death, there was a responsible person for collecting, analyzing, documenting and reporting PHEM data including MDSR data at all level of reporting units. These responsible persons are PHEM Officers, health facility surveillance focal persons, HEWs, HDA) MDSR focal, HMIS focal and RRT committee. Although there was irregular MDSR data analysis at woreda level, regular and detail analysis was performed at sub-city and regional level by PHEM officers, MDSR focal persons and RRT committee. MDSR data was collected, analyzed and reported in daily, weekly and as needed. The data was described epidemiologically by place, person and time that was summarized by table, graph and maps especially at sub-city and region level.

\subsection{Maternal Death Review Committee}

All study units had a maternal death review committee from region to health facility level. The members of the review committees were medical directors, health facility surveillance focal, laboratory expert, pharmacy, senior midwife, $\mathrm{MCH}$ case team, disease prevention and health promotion owner, gynecologist, anesthetist, HEWs focal and HEWs supervisor. They were using maternal death review guide annexed in the MDSR national guideline.

There were 10 community based and 42 facility based maternal death notifications to the region in 2009 EC. This gives case detection rate $13 \%$ of national plan and $130 \%$ of the regional plan for the region. Death review was conducted for all 52 deaths $(100 \%)$ and all notifications were true maternal deaths after verified by regional maternal death review committee. The review committee was able to identify risk factors for 50 (96.15\%) deaths, of which only findings from 7 $(14 \%)$ deaths were used for action or action plan developed. This was due to lack of resource, poor coordination, low attention and follow-up at each level.

Ninety-one percent (91\%) of study units had a separate maternal death preparedness and response plan which was evaluated for last year performance (2009EC).

\subsection{Reports}

Fifty-four percent (54\%) of study units didn't posted the reporting flow chart on the wall of their office. The analysis result of MDSR data was shared to staffs and other stakeholders by presenting it on Technical Working Group (TWG) meeting, printed report, morning session, email and 
phone call. A total of $44(84.6 \%)$ reports were complete with no blank or/and unknown responses at the regional level. The reporting completeness and timeliness of MDSR data at regional level in 2009EC was $98.61 \%$ and $74 \%$ respectively. Sixty-nine percent (69\%) study units believe that the reporting completeness and timeliness can be improved by providing continuous monitoring, feedback, supervision and utilizing electronic based reporting systems like DHIS 2.

\subsection{Training}

Seven (87.5\%) regional PHEM Officers, 18 (90\%) sub-city PHEM officers and 88 (75.2\%) woreda PHEM Officers were trained on PHEM prioritized diseases surveillance system for 13 days and $6(75 \%)$ regional PHEM Officers, 18 (90\%) sub-city PHEM officers and 116 (99.2\%) woreda PHEM Officers were trained on MDSR for 3 days by EPHI. The regional and sub-city health bureaus had also given PHEM disease surveillance training for their staffs. The change after training were not monitored by all levels.

\subsection{Supervision and Feedback}

Irregular supervisions and feedbacks were provided to all sub-cities, woredas and health facilities by the higher level of the government structure. Seventy-seven percent (77\%) and $61.54 \%$ of study units were supervised and received feedback at least one time by the next higher level of governmental structure in 2009EC, respectively. All sub-cities (10) in the city administration was supervised at least one time in the last year (2009 EC) by the region, but no supervision was done from the national. Fifty-four percent (54\%) of study units had conducted at least one-time meeting with the community related to MDSR in the last year (2009EC).

\subsection{Logistics}

All necessary emergency drugs were available and no medical supply shortage was faced at all level of reporting units. Although, there was no separately allocated budget for MDSR system implementation at all level and it is included in general PHEM budget. The system also had got fund from partners such as WHO at regional level. There was also high supply shortage to effectively implement MDSR activities such as printer, vehicle, stationery, fax, email, projector etc.

\subsection{Study Unit's Recommendation/Suggestion}

The following points were suggested by the study units to improve MDSR system implementation:

a) Maintain regular supportive supervision and follow-up by all level government structures.

b) Attract and motivate senior health professionals and partners working on MDSR.

c) Create community awareness on MDSR and change bad community attitudes towards it.

d) Use MDSR data for promotion to attract the attention of leaders and community.

e) Allocate separate MDSR budget and other supplies such as vehicle for MDSR related activities.

f) Provide capacity building trainings for maternal death review committee and untrained staffs including private health facility workers.

g) Use opportunities like trainings and meetings to integrate and strength MDSR activities with other PHEM activities.

h) Strengthen communication and coordination between all level stakeholders on MDSR.

\subsection{Attributes Measurement}

\subsubsection{Simplicity}

Seventy-seven percent (77\%) study units perceive MDSR system was easy and manageable to collect and analyze maternal death data and $92.31 \%$ of study units perceived the case definition of MDSR was easy to be used by all level health workers. Half of the study units believe MDSR system doesn't take much time and have no influence on other activities and $92.31 \%$ believe the system was integrated with other PHEM surveillance systems. Sixty-nine percent (69\%) of perceived that data collection formats used by the system were clear and easy to be filled by all level data collectors. It was also believed MDSR system doesn't need high level training (can be done just by simple orientation) by $15.38 \%$ of study units and $53.85 \%$ of study units believe it was easy to report to the higher level by using available means of reporting systems. Generally, $63.46 \%$ of study units agreed that MDSR system was simple for implementation.

Table 1. Distribution of study units by simplicity measurement for MDSR, Addis Ababa, 2018.

\begin{tabular}{|c|c|c|c|}
\hline Simplicity Measurements & Agree (\%) & Neutral (\%) & Disagree (\%) \\
\hline The data sources of MDSR is easy and manageable & 76.92 & 15.38 & 7.69 \\
\hline The case definition for maternal death is easy to be used by all level health workers & 92.31 & 0.00 & 7.69 \\
\hline MDSR system doesn't take much time and have no influence on other activities & 50.00 & 33.33 & 16.67 \\
\hline The type of data collected are clear and easy & 46.15 & 38.46 & 15.38 \\
\hline The system (MDSR) is integrated with other surveillance systems & 92.31 & 7.69 & 0.00 \\
\hline Data collection formats are clear and easy to be filled by all level data collectors & 69.23 & 7.69 & 23.08 \\
\hline Methods of disseminating MDSR data to whom concerned are easy and manageable & 53.85 & 7.69 & 38.46 \\
\hline
\end{tabular}

\subsubsection{Flexibility and Acceptability}

Fifty-eight percent $(58 \%)$ of study units perceived that
MDSR system was flexible enough to adopt to the user's improvement demands (new case definitions, new data, new technology, new reporting sources etc). 
Thirty-nine percent $(39 \%)$ of study units noticed staffs working on MDSR were satisfied and well accepted the current system. Thirty-one percent of study units identified that health professionals working on MDSR were comfortable when assigned to participate in verbal autopsy activities. The overall acceptability of MDSR system by the health workers working on it was $34.62 \%$.

\subsubsection{Sensitivity and Predictive Value Positive}

Eighty-five percent $(85 \%)$ of study units agreed that the system picks most maternal deaths occurring in the city administration. Sixty-four percent (64\%) of study units had appreciated that most of the deaths (all of the deaths at regional level) reported in the system were actual maternal deaths.

\subsubsection{Representativeness, Timeliness and Cost}

Sixty-seven percent of study units believed that the report of maternal death represents the situation in the city administration. Fifty-eight percent of study units believed MDSR data was submitted on expected time limit. Sixty-nine percent $(69 \%)$ of study units agreed on MDSR system was cost effective compared to the benefit it provides.

\subsubsection{Usefulness}

Seventy-five percent (75\%) of study units agreed the system have an ability to estimate the incidence of maternal deaths and is able to show the trend of maternal deaths in the city administration. Ninety-two percent (92\%) of study units believed that MDSR system had ability to show the progress and effect of preventive and control methods applied to decrease the death of mothers and help the health facilities to improve clinical and ethical practices. It was believed that the system had ability to indicate major causes of maternal deaths in the city administration by $83.33 \%$ of study units and $75 \%$ of study units also believed that MDSR data can stimulate research on maternal death. Sixty-seven percent $(67 \%)$ of study units agreed the data from MDSR can be used for decision making. Generally, $79.85 \%$ of study units agreed that MDSR system was useful if it is implemented as intended.

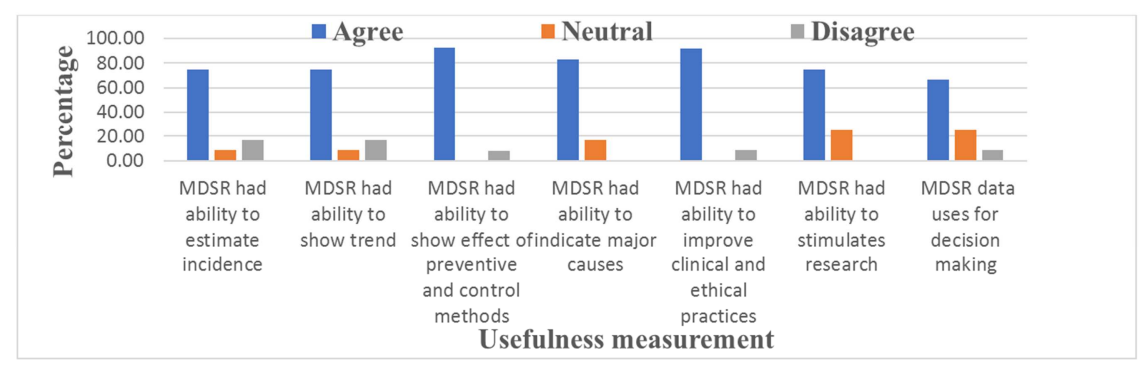

Figure 2. Distribution of study units by usefulness measurement for MDSR, Addis Ababa, 2018.

\subsubsection{Stability}

Seventy-five percent (75\%) of study units agreed that MDSR data was ready for use when needed for planning and other purposes and $16.67 \%$ of study units believe the system was interrupted due to lack of resources within last year (2009EC). The new BPR restructuring had affected the procedures and activities of MDSR as $36.36 \%$ of study units agreed.

\subsubsection{Data Quality}

Seventy-five percent (75\%) of study units agreed MDSR data collection forms were clear and $76.92 \%$ study units agreed most (not specified) MDSR data collectors were trained on MDSR system. Fifty-five percent (55\%) of study units agreed that most of the records were complete. Seventy-three percent $(73 \%)$ of study units agreed that collected data were consistent with the event (maternal death) and $63.64 \%$ of study units agreed the quality of MDSR data was as expected. The overall MDSR data quality in the city administration was $68.57 \%$ and $62.73 \%$ of study units agreed on the overall average attribute measurements of the system.

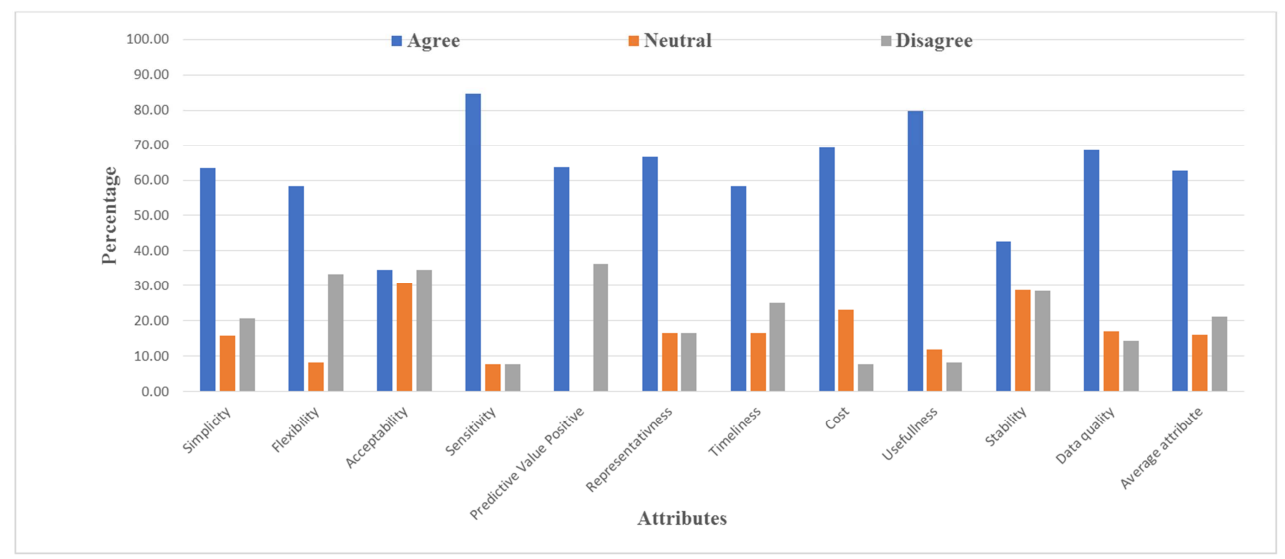

Figure 3. Distribution of study units by attributes measurement for MDSR, Addis Ababa, 2018. 


\section{Discussion}

Sixty-seven percent $(67 \%)$ of study units replied that they had appropriate denominator for MDSR system implementation, but $55.56 \%$ of them didn't put the exact number of the denominator. This might indicate the data quality problem and $33 \%$ of the study units didn't know the denominator used for MDSR. The common type of surveillance used for MDSR in the city administration was comprehensive passive surveillance. The system is relatively young (launched in 2013) and it is not fully strengthened, therefore active surveillance was also used frequently [22].

Most of the regional, sub-city and woreda PHEM Officers were trained on PHEM disease surveillance and MDSR by EPHI. The regional and sub-city health bureaus had also given PHEM disease surveillance training for their staffs including staffs from health facilities. The presence of at least one concerned health professionals almost from all reporting levels were indicated as strength of the training while short period of training, exclusion of private health facilities and MDSR committee from the training were seen as weakness of the training. Additionally, the presence of technical advisors from partners at regional level was seen as an opportunity to share their experience in the training. Although, most of the data collectors at each level were trained on MDSR, some of the current data collectors at community and woreda level were not trained due to high trained staff turnover. Therefore, it might be important to put a minimum duration of time to serve after a training.

Maternal deaths at a health facility were not registered appropriately. This was due to lack of clinical register for maternal deaths and clinical register were available only in $66.67 \%$ of health facilities.

The action threshold used for MDSR in the city administration was one maternal death and $69.23 \%$ of study units replied that they had the capacity to respond for a maternal death within 48 hours. However, this capacity depends on the factors contributing to the death of the mother and timely availability of the information. From the time in which a death of a woman happens to notification, identification of risk factors and development and implementation of action plan takes a minimum of three weeks. Therefore, response to a maternal death will be started after minimum of 21 days if the risk factor for the death of the mother was not identified. Lack of timely notification, poor coordination and communication and shortage of supply were raised as an obstacle for providing timely response.

All of the study units had maternal death review committee from the region to health facility level. Eight percent (8\%) of study units had no separate maternal death review committee but the rapid response team (RRT) is doing all expected activities to be done by the maternal death review committee. The members of the review committees were medical directors, health facility surveillance focal, laboratory expert, pharmacy, senior midwife, $\mathrm{MCH}$ case team, disease prevention and health promotion owner, gynecologist, anesthetist, HEWs focal and HEWs supervisor. These members of the committee may not be similar in profession composition and number of members at each reporting level and site.

There were 10 community based maternal death notifications to the region in $2009 \mathrm{EC}$, of which $4(40 \%)$ of deaths were notified immediately while all deaths were included in the weekly report. There were also 42 facility based maternal death notifications, of which 10 (23.81\%) of the deaths were notified immediately, while all deaths were included in the weekly report. This gives maternal death incidence rate of 4.45 per 100,000 mothers and case detection rate of $13 \%$ of national plan and $130 \%$ of regional plan. Although most of the study units respond that the system picks most maternal deaths, representative and was able to show the trend of maternal deaths in the city administration, it is far from the national plan, case detection rate was too law, not representative and can't show the real maternal death trend of the city administration. However, the original residence for some (not specified) of the died mothers were not Addis Ababa City administration. They came from other regions to seek better treatment and died at a health facility found in Addis Ababa. These deaths were notified to Addis Ababa Regional Health Bureau (AARHB) and communicated to the region they came from to be investigated by the regional health bureau.

Fifty-four percent $(54 \%)$ of study units said that the reporting flow chart were not posted on the wall of their office. Lack of space on the wall of the office and exclusion as performance indicator were raised as a reason for not posting the reporting flow chart. The analysis result of MDSR data was shared to staffs and other stakeholders by presenting it on Technical Working Group (TWG) meeting, printed report, morning session, email and phone call. The regional plan to share the update to stakeholders was every two months but it was not done regularly. Notification to the next higher level was maintained on daily and weekly basis by all level of reporting units. A total of $44(84.6 \%)$ reports were complete with no blank or/and unknown responses at the regional level. In reports which were not complete $(15.4 \%)$, educational status, ethnicity, appropriate code, date of death, age and preventability were commonly missed variables in the report.

Only $63.46 \%$ of study units were agreed MDSR system was simple for implementation and the acceptability of MDSR system by the health workers working on it was $34.62 \%$. These rates are too low to achieve the MDSR establishment goal. The maternal death review committee has a feeling of blaming culture to do maternal death review and this has decreased the system acceptability by the health professionals working on it. Besides this, lack of separate budget and other supplies such as vehicle for MDSR activities decreases staff satisfaction and system acceptability and make system implementation difficult.

Fifty-eight percent (58\%) of study units believed that MDSR data was submitted on expected time limit. The study units had also suggested availing cell phone and air time fee and email 
access help to improve the reporting timeliness. Sixty-nine percent $(69 \%)$ of study units replied that the MDSR system was cost effective compared to the benefit it provides. They also believe this effectiveness will increase if it is implemented as expected with its own budget. Eighty percent (80\%) of study units agreed that MDSR system was useful if it is implemented as intended. They had also suggested use of MDSR data for planning, budget allocation and attracting official's attention, continuous follow-up, feedback and supervision will help to further improve the usefulness of the system. Almost $17 \%$ of study units said the system was interrupted due to lack of resources within last year (2009EC and $36.36 \%$ of study units agreed the new BPR restructuring had affected the procedures and activities of MDSR. This effect might be due to structure change or/and trained staff shift. This indicates the problem of the system in relation to consistency/stability. Since right data enables for appropriate planning and resource mobilization, wrong data causes inappropriate planning and wastage of limited resources. Therefore, the quality of the data collected through MDSR system need to be given high attention. As $68.57 \%$ study units agreed, MDSR data quality in the city administration was as maintained.

The evaluation team had noticed very low motivation and awareness on MDSR among the respondents. This was justified as inconsistent and inappropriate response were given for some of the questions, even they didn't understand after explanation of questions. Respondents had also appointed the evaluation team repeatedly to fill the questioners but without any progress.

Since MDSR system was relatively young (implementation started in 2008EC) it was being implemented in governmental health facilities and almost neglected implementation in private health facilities. Due to this reason the evaluation was done only on governmental health facilities. If private health facilities were included in the evaluation, the result might be totally different from this finding.

\section{Limitation}

Some the respondents had knowledge gap on MDSR and some of the results were not presented numerically. The evaluation excluded private health facilities, which might have a different finding and understanding of this evaluation finding need to consider these limitations.

\section{Conclusion and Recommendation}

The case detection rate of the system was very low and not representative of all maternal deaths occurred in the city administration. The value of system attributes was also very low and it might be impossible to achieve the system objectives unless the value is improved. MDSR system establishment objectives cannot be fulfilled by the current level of implementation.

A separate budget needs to be allocated at all levels of reporting units for MDSR related activities. Capacity building trainings for staffs working on MDSR, regular supervision and feedbacks should be provided continuously by all level of the government structures. It is also critical to monitor the availability of trained staffs and the change after trainings. An agreement might need to be considered before trainings to serve at least for a fixed period of time. Attention should be given to private health facilities in order to achieve the system establishment objective by working coordinately with all stakeholders. The regional MDSR plan needs to be aligned with the national plan.

\section{Acknowledgements}

We would like to acknowledge Ethiopian Public Health Institute for giving permission to evaluate Maternal Death Surveillance and Response system implementation in Addis Ababa City Administration and providing the necessary logistics. Our gratitude also goes to each level health bureau/office PHEM department of Addis Ababa City Administration participated in the study. We also thank Dr. Abiy Girmay and Abigiya Wendmagegnehu for their continuous advice and all individuals who has participated in this MDSR system evaluation process.

\section{References}

[1] Federal Democratic Republic of Ethiopia Public Health Emergency Management. Guidelines for Ethiopia. Ethiopian Health and Nutrition Research Institute Public Health Emergency Management Centre, February 2012, Addis Ababa, Ethiopia.

[2] WHO Regional Committee for Africa: Integrated Diseases Surveillance in the African Region: A Regional Strategy for Communicable Diseases (AFR/RC48/R2). Harare, Zimbabwe, September 1998.

[3] Adokiya MN, Awoonor-Williams JK, Barau IY, Beiersmann C, Mueller O. Evaluation of the integrated disease surveillance and response system for infectious diseases control in northern Ghana. BMC Public Health. 2015; 15: 75.

[4] Pommier de Santi V, Girod R, Mura M, Dia A, Briolant S, Djossou F, et al. Epidemiological and entomological studies of a malaria outbreak among French armed forces deployed at illegal gold mining sites reveal new aspects of the disease's transmission in French Guiana. Malar J. 2016; 15 (1): 35.

[5] World Health Organization, maternal death surveillance and response (MDSR) technical guide, 2013.

[6] World Health Organization, UNICEF. Trends in maternal mortality: 1990-2015: estimates from WHO, UNICEF, UNFPA, World Bank Group and the United Nations Population Division.

[7] Szekeres D. United Nations Millennium Development Goals. Jura: A Pecsi Tudomanyegyetem Allam-es Jogtudomanyi Karanak tudomanyos lapja. 2012: 198.

[8] Central Statistical Agency (CSA) [Ethiopia] and ICF. 2016. Ethiopia Demographic and Health Survey 2016: Key Indicators Report. Addis Ababa, Ethiopia, and Rockville, Maryland, USA CSA and ICF. 
[9] FMOH Ethiopia. Health sector Transformation plan 2015/16-2019/20. MOH Ethiopia, Addis Ababa, 2015.

[10] Demographic and Health Survey (DHS), Ethiopian DHS report, 2011.

[11] WHO, UNICEF, UNFPA and The World Bank, Trends in Maternal Mortality: 1990 to 2013. 2014.

[12] Federal Democratic Republic of Ethiopia, Ministry of Health, Maternal Death Surveillance and Response (MDSR) Technical Guide, 2013.

[13] WHO, Taking Stock Of MATERNAL, NEWBORN and CHILD SURVIVAL 2000-2010 Decade Report, 2011.

[14] WHO, Department of Maternal, Newborn, Child Health and Adolescence Health (MCA), Highlights, 2012-2013 Progress Report, 2014.

[15] Yifru Berhan, Asres Berhan, Causes Of Maternal Mortality In Ethiopia: A Significant Decline In Abortion Related Death, 2013.

[16] Tufh Women and Health Taskforce, Safe Motherhood: Social, Economic, and Medical Determinants of Maternal Mortality, 2006.

[17] Updated Guidelines for Evaluating Public Health Surveillance Systems Recommendations from the Guidelines Working
Group, U.S. Department Of Health And Human Services Centers for Disease Control and Prevention (CDC) Atlanta, GA 303333.

[18] Federal Democratic Republic of Ethiopia Central Statistical Agency. Population Projection of Ethiopia for All Regions at Wereda Level from 2014 - 2017, August 2013, Addis Ababa.

[19] http://populationof2018.com/addis-ababa-population-2018.ht $\mathrm{ml}$

[20] Arscott-Mills S, Holder Y, Gordon G, System JIS. Comparative Evaluation of Different Modes of a National Accident and Emergency Department-Based Injury Surveillance System: Jamaican Experience. Inj Control Saf Promot. 2002 Dec; 9 (4): 235-9.

[21] European Centre For Disease Prevention and Control. Data Quality Monitoring and Surveillance System Evaluation - A Handbook of Methods and Applications. Stockholm: ECDC; 2014.

[22] FMoH Policy Planning Directorate, Health and Health Related Indicators, 2003 E. C (2010/11 G. C).

[23] Baldissera S, Campostrini S, Binkin N, Minardi V, Minelli G, Ferrante G, Et Al. Features And Initial Assessment Of The Italian Behavioral Risk Factor Surveillance System (PASSI), 2007-2008. Prev Chronic Dis. 2011 Jan; 8 (1): A24. 